\title{
Compute methods for assessment of gas distribution networks
}

\author{
L A Kushev \\ Belgorod State Technological University \\ Belgorod, 308012, Russia \\ A Y Feoktistov \\ Belgorod State Technological University \\ Belgorod, 308012, Russia
}

\author{
Y G Ovsyannikov \\ Belgorod State Technological University \\ Belgorod, 308012, Russia \\ M R Kondrasheva \\ Belgorod State Technological University \\ Belgorod, 308012, Russia \\ kondrasheva.mary@yandex.ru
}

\author{
M A Shvydkaya \\ Belgorod State Technological University \\ Belgorod, 308012, Russia
}

\begin{abstract}
The gas industry in the country is the main energy system of the Russian Federation. The main extent of the pipelines unifying the gas supply system (UGSS) is about 700 $000 \mathrm{~km}$. Out of the trunk, transport systems of gas are 150000 $\mathbf{k m}$, and the distribution lines of the gas supply systems are $\mathbf{5 5 0}$ $000 \mathrm{~km}$. The management of functioning and development of these systems propounds various technical and economic tasks which are becoming complicated over the time. Under conditions of the modern economy, the creation of not only highly effective, but also cost reduction systems are required. Therefore the tasks of optimization, together with the mathematical modeling during new devices design and systems become an integral part of the construction process of gas supply systems. The methods improvement of assessment and appearance of high-level performance computers led to creation of a series of program complexes allowing the assessment and solution to engineering design problems.
\end{abstract}

Keywords—component; formatting; style; styling; insert.

\section{INTRODUCTION}

The pipeline systems consist of various pipe lengths/dimensions, diameters and materials that results in additional nonlinear coefficients of hydraulic friction resistance that also needs to be considered, especially when designing long pipe line transport systems under various working environments [1-3]. The configuration of networks, armature has considerable impact on the pressure and uneven distribution of the expenditures. Practically it is possible to locate at least one of the above listed elements in each system.

The program complexes for assessment of distributive systems have begun to develop quickly since the beginning of the $60 \mathrm{~s}$. In this case, the preservation equations in intersections are tackled and Darci-Veysbakh's ratios, Hazen-Williams were applied to definite the pressure losses in communications.
With the computer software development the dimension task solutions of hundreds of thousands of channels and pipelines have become possible using only a personal computer. Currently there are lots of commercial packages, such as CYBERNET, RINCAD, STONER, AQUA, KYPIPE, Camp Dresser \& McKee, CalcFlow and Piccolo.

One of leaders of commercial packages domestically produced for assessment of hydraulic networks is Hydraulic Complex System program, NTP "Pipeline". Also such domestic software products as Data Processing Centers (DPC) "Stream", ZULU "GAZ" are used for modelling of multi networks [4].

\section{MATERIALS AND METHODS}

Generally, the problem of hydraulic assessment is concentrated on the determination of flow (and diameters) sites of the pipeline distribution system according to at specific load duties taking into account technological and economic restrictions [5]. The evaluated parameters are gas streams on the system sites of the gas distribution pipelines, the differences of pressure on settlement sites and the pressure in the different intersections of distribution system of gas supply.

The basic data for assessment are the description of geometrical structure of the gas system supply, diameters, lengths and material of sites of gas pipelines, output pressure and productivity of natural gas sources, expenditures involving consumers and low pressure transportation expenditures and also the parameters defining the transported natural gas properties. Hydraulic assessments of gas distribution networks are carried out on the basis of the general equations of gas dynamics establishing a link between the diameter of the pipeline, gas flow and pressure difference for the section of the pipeline of specific length and design, 
and mathematical methods providing solution to the hydraulic problem assessment with exact accuracy.

The program of hydraulic assessment which is Hydraulic Calculator is intended for assessment of the established stream of gas supply distributive systems of high, medium or low pressure. With the help of the program it is possible to assess practically any systems of gas distribution pipelines from various materials and to analyse their work functioning status [6].

\section{MAIN PART}

Designing gas pipelines, especially under the conditions of huge distance and wide range branches the hydraulic assessment by means selection and specification of necessary diameters is made for economic transportation of gas is made. The deadlock and ring gas networks of low, medium and high pressure functioning from one or several GRP, GRS are subjected to assessment. The assessment of gas supply systems can be made using two techniques: taking into account gas temperature under normal working conditions on the SP 42-101-2003; taking into account gas difference temperature under normal conditions according to the requirements of STO GP GR 12.2.2-1-2013.

A mathematical imitation model (fig. 1) of gas supply system has been considered from the example of the assessment scheme of an operating mode of gas pipeline of high pressure. This serves for the solution of a testing task which allows the analysis of hydraulic operating modal system and also to predict its changes in case of any deviations. The purpose of assessment testing is to determine the actual gas usage along the gas networks pressure in all period points and violations of consumer modal operation [7]. The assessments can be carried out at various initial basic data, including at a shutdown of certain gas network areas. In each area of network, from a starting point number of the area, to a completing point number area, area dimension in meters, the gas consumption is specified in the usage assessment scheme. The point 0 is input, points $1-6$ are the consumer line connections.

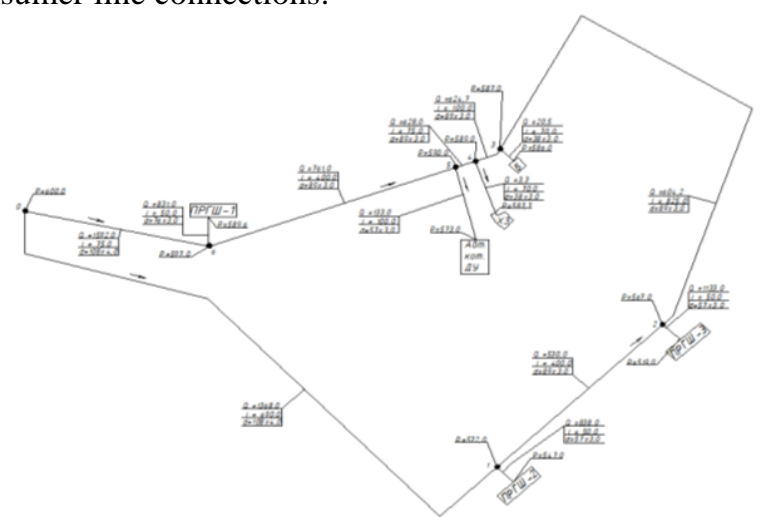

Fig. 1. Assessment scheme of gas pipeline modal operation of high pressure of the micro-district Near-Igumenka-1

Due to short temporary emergencies, some decline in the system can be envisaged, which is evaluated by the security coefficient, depending on the consumer category. In emergency cases, the supervision service takes measures for gas consumption control. This brings about the reduction in the heat supply to buildings and hot water supply that will lead to a decrease in the gas consumption in boiler rooms; temporary work cessation of secondary workshops; transfer of reserve from gas to other type of fuel in the boiler rooms and shops. However in all cases, the pressure mode in the gas network has to ensure normal functioning of gas burner devices and units.

For one gas network ring, it is necessary to forecast two emergency situations: a shutdown of the main section on the left and right of energy source. Since the gas pipeline ring turns in to a deadlock, the diameters of sections of the ring can be determined by limited gas supply to consumers through the deadlock line rates. A network of high pressure analysis on the program of hydraulic assessment is made by Hydraulic Calculator. Assessment schemes of emergency shutdown operation of the most loaded sections are 0-1 and 0-6.

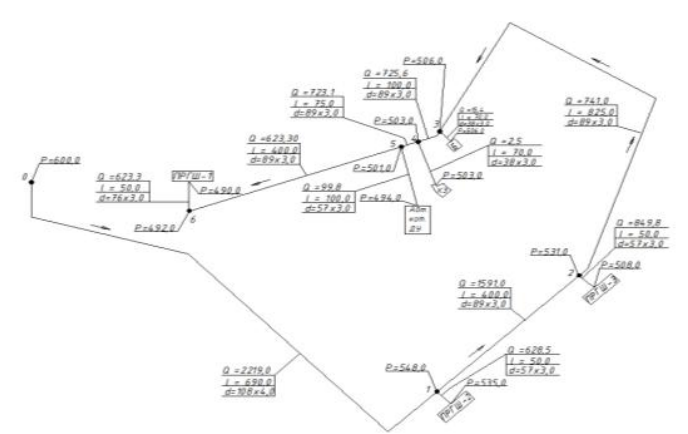

Fig. 2. Assessment scheme of emergency shutdown operation of the high pressure gas pipeline of section $0-6$ of the micro-district has been submitted Near-Igumenka-1

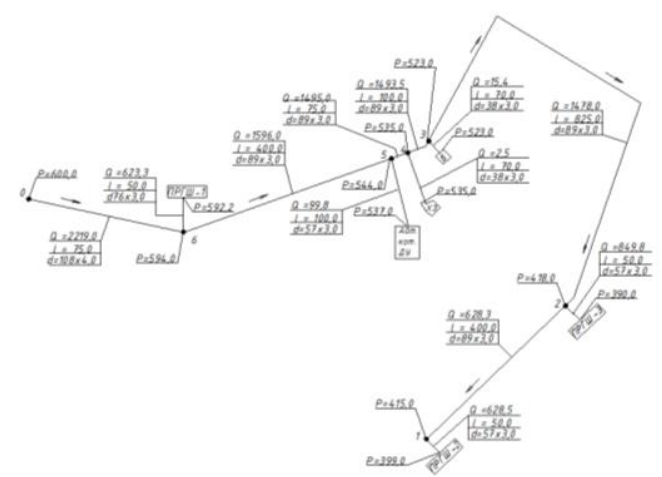

Fig. 3. Assessment scheme of emergency shutdown operation of the highpressure gas pipeline of section $0-1$ of the micro-district has been submitted by Near-Igumenka-1.

Pressure on the point 0 (at the exit from GRP) of $600 \mathrm{kPa}$ is the greatest possible category for (the high pressure gas pipeline) of 2ndcategory. The final values in the high pressure pipeline is considered $50 \mathrm{kPa}$ which is above the initial pressure of subsequent categories $-350 \mathrm{kPa}$, according to [5, 8] which doesn't exceed the extreme value at emergency operation (tab. 1). Considering the range of admissible pressure, the final dis-balance value will be $42 \%$. 
TABLE I. ASSESSMENT OF EMERGENCY OPERATION OF THE HIGH PRESSURE GAS PIPELINE

\begin{tabular}{|c|c|c|c|c|}
\hline Subscriber & $\begin{array}{l}\text { Distance } \\
\text { from a } \\
\text { supply } \\
\text { point to } \\
\text { the } \\
\text { subscriber } \\
\mathrm{L}, \mathrm{M}\end{array}$ & $\begin{array}{l}\text { Assessment } \\
\text { pressure } \\
\text { PP, kPa }\end{array}$ & $\begin{array}{l}\text { Pressure } \\
\text { at } \\
\text { emergency } \\
\text { operation } \\
\text { PaB, kPa }\end{array}$ & $\begin{array}{l}\text { Pressure } \\
\text { disbalance, } \\
\%\end{array}$ \\
\hline \multicolumn{5}{|c|}{ Rejection of lot/plot 0-6 } \\
\hline 1 & 690 & 572 & 548 & 4 \\
\hline 2 & 1090 & 567 & 531 & 6 \\
\hline 3 & 1915 & 587 & 506 & 14 \\
\hline 4 & 2015 & 589 & 503 & 15 \\
\hline 5 & 2090 & 590 & 501 & 16 \\
\hline 6 & 2490 & 597 & 492 & 18 \\
\hline \multicolumn{5}{|c|}{ Rejection of lot/plot 0-6 0-1 } \\
\hline 6 & 75 & 597 & 594 & 0,5 \\
\hline 5 & 475 & 590 & 544 & 8 \\
\hline 4 & 550 & 589 & 535 & 9 \\
\hline 3 & 650 & 587 & 523 & 10 \\
\hline 2 & 1475 & 567 & 418 & 20 \\
\hline 1 & 1875 & 572 & 415 & 22 \\
\hline
\end{tabular}

Analyzing the disbalance of pressure, one can notice the increase in its non-uniformity. In case of emergency shutdown of section $0-6$, the subscriber has $3 \%$ increase which is more than twice. This is connected with the fact that in the operational mode, subscribers 3-6 are not the final, in emergency operation, gas moves in the opposite direction. In case of failure of section $0-1$, one can observe non-uniformity in increase in dis-balance of subscribers 5 and 2 .

\section{CONCLUSION}

Computer methods of assessment of gas-distribution networks are one of the main methods of assessment. This assessment confirms that the diameter selection of gas pipeline corresponds to $[5,8,9]$. Thus, to determine distribution pressure, values and the directions of flows in the gas supply systems at a designing stage is the key for reliable and overall performance of systems. The high level of reliability of gas flow can be provided only in the case of new design method applications and constructions. Therefore the tasks of optimization, together with mathematical imitation in case of design of new devices and systems, have become an integral part of the design process of gas-distribution systems. The computer assessment method of gas systems allows one to increase the efficiency of design systems taking into account the influence of design and operational conditions and also, to reduce periods of design at the expense of the possibility of modification of constructive diagram systems at all designing stages.

\section{ACKNOWLEDGEMENT}

The article was prepared within the development program of the Flagship Regional University on the basis of Belgorod State Technological University named after V.G. Shoukhov, using equipment of High Technology Center at BSTU named after V.G. Shoukhov.

\section{References}

[1] A. A. Ionin, V. A. Zhila, V. V. Artikhovich, M. G. Pshonik, Gas supply: the textbook for students of higher educational institutions as "Gas power supply and ventilation". Moscow: DIA publishing house, 2012.

[2] L. A. Kushev, G. L. Okuneva, D. Y. Suslov, A. A. Gravin, Chemical and petroleum engineering, Vol. 9-10, pp. 613-618, 2012.

[3] D. Yu. Suslov, G. K. Vyrodov, Bulletin of the BSTU named after V G Shoukhov, Vol. 10, pp. 114-119, 2016.

[4] V. Yu. Volkov, Development of assessment method of difficult networks of pneumatic systems: Candidate of Tech.Sci., Moscow, 2015.

[5] Joint venture 62.13330.2011. Set of rules. Gasdistributing systems. The staticized editorial office Construction Norms and Regulations 42-012002.

[6] JSC Giproniigaz "Hydraulic Assessment", User's guide, Saratov.

[7] GIS Zulu [Electronic Resource] of URL: http://www.geoinfograd.ru/zulu.htm (Date submitted: 10/11/2017)

[8] Joint venture 42-101-2003 General provisions for designing and construction of gas distributing systems from metal to polyethylene pipes.

[9] Joint venture 42-102-2004 Designing and construction of gas pipelines from metal pieces.

[10] G. G. Nasr, N. E. Connor, Natural Gas Engineering and Safety Challenges, London, 2014.

[11] Yu. B. Gritsenko, Yu. P. Ekhlakov, O. I. Zhukovsky, Geo-information technologies for monitoring engineering networks: monograph Tomsk: Tomsk publishing house, State University that control systems and radio electronics, 2010. 